\title{
F-cone metric spaces over Banach algebra
}

Jerolina Fernandez' , Neeraj Malviya', Stojan Radenovič ${ }^{2,3^{*}}$ and Kalpana Saxena ${ }^{4}$

"Correspondence:

stojan.radenovic@tdt.edu.vn

${ }^{2}$ Nonlinear Analysis Research Group,

Ton Duc Thang University, Ho Chi

Minh City, Vietnam

${ }^{3}$ Faculty of Mathematics and

Statistics, Ton Duc Thang University,

Ho Chi Minh City, Vietnam

Full list of author information is

available at the end of the article

\section{然 Springer}

\begin{abstract}
The paper deals with the achievement of introducing the notion of F-cone metric spaces over Banach algebra as a generalization of $N_{p}$-cone metric space over Banach algebra and $\mathrm{N}_{b}$-cone metric space over Banach algebra and studying some of its topological properties. Also, here we define generalized Lipschitz and expansive maps for such spaces. Moreover, we investigate some fixed points for mappings satisfying such conditions in the new framework. Subsequently, as an application of our results, we provide an example. Our results generalize some well-known results in the literature.

MSC: 46B20; 46B40; 46J10; 54A05; 47H10

Keywords: F-cone metric space over Banach algebra; c-sequence; generalized Lipschitz mapping; expansive mapping; fixed point
\end{abstract}

\section{Introduction}

Partial metric spaces were introduced by Matthews [1] in 1994. He studied a partial metric space as a part of the denotational semantics of dataflow networks and showed that the Banach contraction principle can be generalized to the partial metric context for applications in program verification. Especially, it has the property that differentiates it from other spaces, that is, the self-distance of any point may not be zero, also a convergent sequence need not have unique limit in these spaces.

On the other hand, in 1989, the concept of $b$-metric spaces was introduced by Bakhtin [2] as a generalization of metric spaces. He showed the contraction mapping principle in a $b$-metric space that generalizes the prominent Banach contraction principle in metric spaces.

In the same spirit, recently, Huang and Zhang [3] replaced the set of real numbers by ordering Banach space and defined a cone metric space as a generalization of the metric space. The authors proved some fixed point theorems of contractive mappings on cone metric spaces. They also defined the Cauchy sequence and convergence of a sequence in such spaces in terms of interior points of the underlying cone. After that, in [4], Rezapour and Hamlbarani generalized some results of [5] by omitting the assumption of normality. For fixed point theorems on cone metric spaces, readers may refer to [6-9] and the references therein.

Malviya et al. [10] introduced the concept of $N$-cone metric spaces, which is a new generalization of the generalized G-cone metric space [11] and the generalized $D^{*}$-metric space [12]. They proved fixed point theorems for asymptotically regular maps and se-

(c) The Author(s) 2017. This article is distributed under the terms of the Creative Commons Attribution 4.0 International License (http://creativecommons.org/licenses/by/4.0/), which permits unrestricted use, distribution, and reproduction in any medium, provided you give appropriate credit to the original author(s) and the source, provide a link to the Creative Commons license, and indicate if changes were made. 
quences. Afterwards, in [13], the authors defined contractive maps in $N$-cone metric spaces and proved various fixed point theorems for such maps.

Despite these features, some authors demonstrated that the fixed point results proved on cone metric spaces are the straightforward outcome of the corresponding results of usual metric spaces where the real-valued metric function $d^{*}$ is defined by a nonlinear scalarization function $\xi_{e}$ (see [14]) or by a Minkowski functional $q_{e}$ (see [5]).

Due to the concrete reasons mentioned above, researchers were losing their interest in a cone metric space. Fortunately, Liu and Xu [15] introduced the approach of cone metric spaces over Banach algebras by replacing Banach spaces $E$ by Banach algebras $A$ as the underlying spaces of cone metric spaces. They verified some fixed point theorems of generalized Lipschitz mappings with weaker conditions on the generalized Lipschitz constant $k$ by means of the spectral radius. Not long ago, Xu and Radenović [16] deleted the normality of cones and greatly generalized the main results of [15]. In particular, some authors have shown recently some fixed point results given in [17-19].

Following these ideas, very recently, Fernandez et al. [6] introduced the notion of partial cone metric spaces over Banach algebra as a generalization of partial metric spaces and cone metric spaces over Banach algebra, which was selected for Young Scientist Award 2016, M.P., India (see [20]).

Recently, proceeding in this direction, Fernandez et al. introduced the structure of $N_{p^{-}}$ cone metric space over Banach algebra [21] as a generalization of $N$-cone metric space over Banach algebra [22] and partial metric space and $N_{b}$-cone metric space over Banach algebra [23] as a generalization of $N$-cone metric space over Banach algebra [22] and $b$ metric space, respectively.

Inspired and encouraged by the previous works, we present seven sections in this paper. For the reader's convenience, we recall in Section 2 some definitions that will be used in the sequel. In Section 3, after the preliminaries, we introduce $F$-cone metric spaces over Banach algebra which generalize $N_{p}$-cone metric spaces over Banach algebra and $N_{b}$-cone metric spaces over Banach algebra. In Section 4, we discuss the topological properties. In Section 5, we introduce the notions of generalized Lipschitz and expansive maps. Section 6 is devoted to deriving the existence of fixed point theorems for such spaces by using the mentioned contractive conditions. Finally, in Section 7, we define expansive maps and investigate the existence and uniqueness of the fixed point in the new framework. Our main theorems extend and unify existing results in the recent literature. We also give illustrative examples that verify our results.

\section{Preliminaries}

We begin with the following definition as a recall from [15].

Let $A$ always be a real Banach algebra. That is, $A$ is a real Banach space in which an operation of multiplication is defined subject to the following properties (for all $x, y, z \in A$, $\alpha \in R)$ :

1. $(x y) z=x(y z)$;

2. $x(y+z)=x y+x z$ and $(x+y) z=x z+y z$;

3. $\alpha(x y)=(\alpha x) y=x(\alpha y)$;

4. $\|x y\| \leq\|x\|\|y\|$.

Throughout this paper, we shall assume that a Banach algebra has a unit (i.e., a multiplicative identity) $e$ such that $e x=x e=x$ for all $x \in A$. An element $x \in A$ is said to be 
invertible if there is an inverse element $y \in A$ such that $x y=y x=e$. The inverse of $x$ is denoted by $x^{-1}$. For more details, we refer the reader to [24].

The following proposition is given in [24].

Proposition 2.1 Let $A$ be a Banach algebra with a unit e, and $x \in A$. If the spectral radius $\rho(x)$ of $x$ is less than 1 , i.e.,

$$
\rho(x)=\lim _{n \rightarrow \infty}\left\|x^{n}\right\|^{\frac{1}{n}}=\inf \left\|x^{n}\right\|^{\frac{1}{n}}<1,
$$

then $e-x$ is invertible. Actually,

$$
(e-x)^{-1}=\sum_{i=0}^{\infty} x^{i}
$$

Remark 2.2 From [24] we see that the spectral radius $\rho(x)$ of $x$ satisfies $\rho(x) \leq\|x\|$ for all $x \in A$, where $A$ is a Banach algebra with a unit $e$.

Remark 2.3 (see [16]) In Proposition 2.1, if the condition ' $\rho(x)<1^{\prime}$ is replaced by $\|x\| \leq 1$, then the conclusion remains true.

Remark 2.4 (see [16]) If $\rho(x)<1$, then $\|x\|^{n} \rightarrow 0(n \rightarrow \infty)$.

Lemma 2.5 (see [25]) If $E$ is a real Banach space with a solid cone $P$ and if $\theta \preccurlyeq u \ll c$ for each $\theta \ll c$, then $u=\theta$.

A subset $P$ of $A$ is called a cone of $A$ if

1. $P$ is nonempty closed and $\{\theta, e\} \subset P$;

2. $\alpha P+\beta P \subset P$ for all nonnegative real numbers $\alpha, \beta$;

3. $P^{2}=P P \subset P$

4. $P \cap(-P)=\{\theta\}$,

where $\theta$ denotes the null of the Banach algebra $A$. For a given cone $P \subset A$, we can define a partial ordering $\preccurlyeq$ with respect to $P$ by $x \preccurlyeq y$ if and only if $y-x \in P$. $x \prec y$ will stand for $x \preccurlyeq y$ and $x \neq y$, while $x \ll y$ will stand for $y-x \in \operatorname{int} P$, where int $P$ denotes the interior of $P$. If int $P \neq \varnothing$, then $P$ is called a solid cone.

The cone $P$ is called normal if there is a number $M>0$ such that, for all $x, y \in A$,

$$
\theta \preccurlyeq x \preccurlyeq y \quad \Rightarrow \quad\|x\| \leq M\|y\| .
$$

The least positive number satisfying the above is called the normal constant of $P$ [3].

In the following we always assume that $A$ is a Banach algebra with a unit $e, P$ is a solid cone in $A$ and $\preccurlyeq$ is the partial ordering with respect to $P$.

Definition $2.6([3,15])$ Let $X$ be a nonempty set. Suppose that the mapping $d: X \times X \rightarrow A$ satisfies

1. $\theta \prec d(x, y)$ for all $x, y \in X$ and $d(x, y)=\theta$ if and only if $x=y$;

2. $\quad d(x, y)=d(y, x)$ for all $x, y \in X$;

3. $d(x, y) \preccurlyeq d(x, z)+d(z, y)$ for all $x, y, z \in X$. 
Then $d$ is called a cone metric on $X$, and $(X, d)$ is called a cone metric space over Banach algebra $A$.

For other definitions and related results on cone metric space over Banach algebra, we refer to [15].

Definition 2.7 ([2]) Let $X$ be a nonempty set and $s \geq 1$ be a given real number. A function $d: X \times X \rightarrow R^{+}$is a $b$-metric on $X$ if, for all $x, y, z \in X$, the following conditions hold:

1. $d(x, y)=0$ if and only if $x=y$;

2. $\quad d(x, y)=d(y, x)$;

3. $d(x, z) \leq s[d(x, y)+d(y, z)]$.

In this case, the pair $(X, d)$ is called a $b$-metric space.

For more definitions and results on $b$-metric spaces, we refer the reader to [26].

Definition 2.8 ([1]) A partial metric on a nonempty set $X$ is a function $p: X \times X \rightarrow R^{+}$ such that for all $x, y, z \in X$, the following conditions hold:

1. $x=y \Leftrightarrow p(x, x)=p(x, y)=p(y, y)$;

2. $p(x, x) \leq p(x, y)$;

3. $p(x, y)=p(y, x)$;

4. $p(x, y) \leq p(x, z)+p(z, y)-p(z, z)$.

The pair $(X, p)$ is called a partial metric space. It is clear that if $p(x, y)=0$, then from (1) and (2) $x=y$. But if $x=y, p(x, y)$ may not be 0 .

Definition 2.9 ([10]) Let $X$ be a nonempty set. A function $N: X^{3} \rightarrow A$ is called $N$-cone metric on $X$ if for any $x, y, z, a \in X$, the following conditions hold:

$\left(N_{1}\right) \quad 0 \leq N(x, x, x)$

$\left(N_{2}\right) \quad N(x, y, z)=\theta$ iff $x=y=z$;

$\left(N_{3}\right) N(x, y, z) \leq N(x, x, a)+N(y, y, a)+N(z, z, a)$.

Then the pair $(X, N)$ is called an $N$-cone metric space over Banach algebra $A$.

Definition 2.10 ([23]) An $N_{b}$-cone metric on a nonempty set $X$ is a function $N_{b}: X^{3} \rightarrow A$ such that for all $x, y, z, a \in X$ :

$\left(N_{b_{1}}\right) \quad \theta \preccurlyeq N_{b}(x, y, z)$;

$\left(N_{b_{2}}\right) N_{b}(x, y, z)=\theta$ iff $x=y=z$;

$\left(N_{b_{3}}\right) N_{b}(x, y, z) \preccurlyeq s\left[N_{b}(x, x, a)+N_{b}(y, y, a)+N_{b}(z, z, a)\right]$.

The pair $\left(X, N_{b}\right)$ is called an $N_{b}$-cone metric space over Banach algebra $A$. The number $s \geq 1$ is called the coefficient of $\left(X, N_{b}\right)$.

Definition 2.11 ([21]) An $N_{p}$-cone metric on a nonempty set $X$ is a function $N_{p}: X^{3} \rightarrow A$ such that for all $x, y, z, a \in X$ :

$\left(N_{p} 1\right) \quad x=y=z \Leftrightarrow N_{p}(x, x, x)=N_{p}(y, y, y)=N_{p}(z, z, z)=N_{p}(x, y, z) ;$

$\left(N_{p} 2\right) \theta \preccurlyeq N_{p}(x, x, x) \preccurlyeq N_{p}(x, x, y) \preccurlyeq N_{p}(x, y, z)$, for all $x, y, z \in X$ with $x \neq y \neq z$;

$\left(N_{p} 3\right) N_{p}(x, y, z) \preccurlyeq N_{p}(x, x, a)+N_{p}(y, y, a)+N_{p}(z, z, a)-N_{p}(a, a, a)$.

The pair $\left(X, N_{p}\right)$ is called an $N_{p}$-cone metric space over Banach algebra $A$. 


\section{F-cone metric space over Banach algebra}

In this section, we define a new structure, i.e., $F$-cone metric spaces over Banach algebra.

Definition 3.1 Let $X$ be a nonempty set. A function $F: X^{3} \rightarrow A$ is called $F$-cone metric on $X$ if for any $x, y, z, a \in X$, the following conditions hold:

$\left(F_{1}\right) \quad x=y=z$ iff $F(x, x, x)=F(y, y, y)=F(z, z, z)=F(x, y, z)$;

$\left(F_{2}\right) \quad \theta \preccurlyeq F(x, x, x) \preccurlyeq F(x, x, y) \preccurlyeq F(x, y, z)$, for all $x, y, z \in X$ with $x \neq y \neq z$;

$\left(F_{3}\right) F(x, y, z) \preccurlyeq s[F(x, x, a)+F(y, y, a)+F(z, z, a)]-F(a, a, a)$.

Then the pair $(X, F)$ is called an $F$-cone metric space over Banach algebra $A$. The number $s \geq 1$ is called the coefficient of $(X, F)$.

Remark 3.2 In an $F$-cone metric space over Banach algebra $(X, F)$, if $x, y, z \in X$ and $F(x, y, z)=\theta$, then $x=y=z$, but the converse may not be true.

Example 3.3 Let $A=C_{1}^{R}[0,1]$ and define a norm on $A$ by $\|x\|=\|x\|_{\infty}+\left\|x^{\prime}\right\|_{\infty}$ for $x \in A$. Define multiplication in $A$ as just pointwise multiplication. Then $A$ is a real unit Banach algebra with unit $e=1$. Set $P=\{x \in A: x \geq 0\}$ is a cone in $A$. Moreover, $P$ is not normal (see [4]). Let $X=[0, \infty)$. Define a mapping $F: X^{3} \rightarrow A$ by $F(x, y, z)(t)=\left((\max \{x, z\})^{2}+\right.$ $\left.(\max \{y, z\})^{2},(\max \{x, z\})^{2}+(\max \{y, z\})^{2}\right) e^{t}$ for all $x, y, z \in X$, and let $\alpha>0$ be any constant. Then $(X, F)$ is an $F$-cone metric space over Banach algebra $A$ with the coefficient $s=2$. But it is not an $N_{p}$-cone metric space over Banach algebra since the triangle inequality is not satisfied; neither it is an $N_{b}$-cone metric space over Banach algebra $A$ since for any $x>0$, we have $N_{b}(x, x, x)(t)=2 x^{2} \cdot e^{t} \neq \theta$.

Lemma 3.4 Let $(X, F)$ be an F-cone metric space over Banach algebra $A$. Then

(a) if $F(x, y, z)=\theta$, then $x=y=z$.

(b) if $x \neq y$, then $F(x, x, y)>\theta$.

Proof The proof is obvious.

Proposition 3.5 If $(X, F)$ is an F-cone metric space over Banach algebra, then for all $x, y, z \in X$, we have $F(x, x, y)=F(y, y, x)$.

Definition 3.6 Let $(X, F)$ be an $F$-cone metric space over Banach algebra $A$. Then, for $x \in X$ and $c>\theta$, the $F$-balls with center $x$ and radius $c>\theta$ are

$$
B_{F}(x, c)=\{y \in X: F(x, x, y) \ll F(x, x, x)+c\} .
$$

\section{Topology on $F$-cone metric space over Banach algebra}

In this section, we define the topology of $F$-cone metric space over Banach algebra and study its topological properties.

Definition 4.1 Let $(X, F)$ be an $F$-cone metric space over Banach algebra $A$ with coefficient $s \geq 1$. For each $x \in X$ and each $\theta \ll c$, put $B_{F}(x, c)=\{y \in X: F(x, x, y) \ll F(x, x, x)+c\}$ and put $B=\left\{B_{F}(x, c): x \in X\right.$ and $\left.\theta \ll c\right\}$. Then $B$ is a subbase for some topology $\tau$ on $X$. 
Remark 4.2 Let $(X, F)$ be an $F$-cone metric space over Banach algebra $A$. In this paper, $\tau$ denotes the topology on $X, B$ denotes a subbase for the topology on $\tau$ and $B_{F}(x, c)$ denotes the $F$-ball in $(X, F)$, which are described in Definition 4.1. In addition, $U$ denotes the base generated by the subbase $B$.

Theorem 4.3 Let $(X, F)$ be an F-cone metric space over Banach algebra $A$, and let $P$ be a solid cone in $A$. Let $k \in P$ be an arbitrarily given vector, then $(X, F)$ is a Hausdorff space.

Proof Let $(X, F)$ be an $F$-cone metric space over Banach algebra, and let $x, y \in X$ with $x \neq y$. Let $F(x, x, y)=c$.

Suppose $U=B\left(x, \frac{c}{3}\right)$ and $V=B\left(y, \frac{c}{3}\right)$.

Then $x \in U$ and $y \in V$.

We claim that $U \cap V=\phi$.

If not, there exists $z \in U \cap V$.

But then

$$
F(x, x, z) \prec \frac{c}{3 s} \quad \text { and } \quad F(y, y, z) \prec \frac{c}{3 s} .
$$

So, we get

$$
\begin{aligned}
c=F(x, x, y) & \preccurlyeq s[F(x, x, z)+F(x, x, z)+F(y, y, z)]-F(z, z, z) \\
& \prec s[2 F(x, x, z)+F(y, y, z)] \\
& \preccurlyeq s\left[\frac{2 c}{3 s}+\frac{c}{3 s}\right] \\
& \prec c,
\end{aligned}
$$

i.e., $c<c$, which is a contradiction.

Hence $U \cap V=\phi$ and $X$ is a Hausdorff space.

Now, we define $\theta$-Cauchy sequence and convergent sequence in an $F$-cone metric space over Banach algebra $A$.

Definition 4.4 Let $(X, F)$ be an $F$-cone metric space over Banach algebra $A$. A sequence $\left\{x_{n}\right\}$ in $(X, F)$ converges to a point $x \in X$ whenever for every $c \gg \theta$ there is a natural number $N$ such that $F\left(x_{n}, x, x\right) \ll c$ for all $n \geq N$. We denote this by

$$
\lim _{n \rightarrow \infty} x_{n}=x \quad \text { or } \quad x_{n} \rightarrow x \quad(n \rightarrow \infty)
$$

Definition 4.5 Let $(X, F)$ be an $F$-cone metric space over Banach algebra $A$. A sequence $\left\{x_{n}\right\}$ in $X$ is said to be a $\theta$-Cauchy sequence in $(X, F)$ if $\left\{F\left(x_{n}, x_{m}, x_{m}\right)\right\}$ is a $c$-sequence in $A$, i.e., if for every $c \gg \theta$ there exists $n_{0} \in N$ such that $F\left(x_{n}, x_{m}, x_{m}\right) \ll c$ for all $n, m \geq n_{0}$.

Definition 4.6 Let $(X, F)$ be an $F$-cone metric space over Banach algebra $A$. Then $X$ is said to be $\theta$-complete if every $\theta$-Cauchy sequence $\left\{x_{n}\right\}$ in $(X, F)$ converges to $x \in X$ such that $F(x, x, x)=\theta$. 
Definition 4.7 Let $(X, F)$ and $\left(X^{\prime}, F^{\prime}\right)$ be an $F$-cone metric space over Banach algebra $A$. Then a function $f: X \rightarrow X^{\prime}$ is said to be continuous at a point $x \in X$ if and only if it is sequentially continuous at $x$, that is, whenever $\left\{x_{n}\right\}$ is convergent to $x$, we have $\left\{x_{n}\right\}$ is convergent to $f(x)$.

\section{Generalized Lipschitz maps}

In this section, we define generalized Lipschitz maps in $F$-cone metric spaces over Banach algebra.

Definition 5.1 Let $(X, F)$ be an $F$-cone metric space over Banach algebra $A$ and $P$ be a cone in $A$. A map $T: X \rightarrow X$ is said to be a generalized Lipschitz mapping if there exists a vector $k \in P$ with $\rho(k)<1$ for all $x, y \in X$ such that

$$
F(T x, T x, T y) \preccurlyeq k F(x, x, y) .
$$

Example 5.2 Let the Banach algebra $A$ and the cone $P$ be the same ones as those in Example 3.3, and let $X=R^{+}$. Define a mapping $F: X^{3} \rightarrow A$ as in Example 3.3. Then $(X, F)$ is an $F$-cone metric space over Banach algebra $A$. Now define the mapping $T: X \rightarrow X$ by $T(x)=\frac{x}{3} \cos \frac{x}{3}$. Since $u \cos u \leq u$ for each $u \in[0, \infty)$, for all $x, y \in X$, we have

$$
\begin{aligned}
F & (T x, T x, T y) \\
& =\left((\max \{T x, T y\})^{2}+(\max \{T x, T y\})^{2}, \alpha\left((\max \{T x, T y\})^{2}+(\max \{T x, T y\})^{2}\right)\right) \cdot e^{t} \\
& =2\left[(\max \{T x, T y\})^{2}, \alpha(\max \{T x, T y\})^{2}\right] \cdot e^{t} \\
& =2\left[\left(\max \left\{\frac{x}{3} \cos \frac{x}{3}, \frac{y}{3} \cos \frac{y}{3}\right\}\right)^{2}, \alpha\left(\max \left\{\frac{x}{3} \cos \frac{x}{3}, \frac{y}{3} \cos \frac{y}{3}\right\}\right)^{2}\right] \cdot e^{t} \\
& \preccurlyeq 2\left[\left(\max \left\{\frac{x}{3}, \frac{y}{3}\right\}\right)^{2}, \alpha\left(\max \left\{\frac{x}{3}, \frac{y}{3}\right\}\right)^{2}\right] \cdot e^{t} \\
& \preccurlyeq \frac{2}{9}\left[(\max \{x, y\})^{2}, \alpha(\max \{x, y\})^{2}\right] \cdot e^{t} \\
& =\frac{1}{9}\left[(\max \{x, y\})^{2}+(\max \{x, y\})^{2}, \alpha\left((\max \{x, y\})^{2}+(\max \{x, y\})^{2}\right)\right] \cdot e^{t} \\
& \preccurlyeq \frac{1}{9} F(x, x, y)(t),
\end{aligned}
$$

where $k=\frac{1}{9}$. Clearly, $T$ is a generalized Lipschitz map in $X$.

Now we review some facts on $c$-sequence theory.

Definition 5.3 ([27]) Let $P$ be a solid cone in a Banach space $E$. A sequence $\left\{u_{n}\right\} \subset P$ is said to be a $c$-sequence if for each $c \gg \theta$ there exists a natural number $N$ such that $u_{n} \ll c$ for all $n>N$.

Lemma 5.4 ([28]) If $E$ is a real Banach space with a solid cone $P$ and $\left\{u_{n}\right\} \subset P$ is a sequence with $\left\|u_{n}\right\| \rightarrow 0(n \rightarrow \infty)$, then $u_{n}$ is a c-sequence. 
Lemma 5.5 ([24]) Let $A$ be a Banach algebra with a unit $e, k \in A$, then $\lim _{n \rightarrow \infty}\left\|k^{n}\right\|^{\frac{1}{n}}$ exists and the spectral radius $\rho(k)$ satisfies

$$
\rho(k)=\lim _{n \rightarrow \infty}\left\|k^{n}\right\|^{\frac{1}{n}}=\inf \left\|k^{n}\right\|^{\frac{1}{n}}
$$

If $\rho(k)<|\lambda|$, then $(\lambda e-k)$ is invertible in $A$; moreover,

$$
(\lambda e-k)^{-1}=\sum_{i=0}^{\infty} \frac{k^{i}}{\lambda^{i+1}},
$$

where $\lambda$ is a complex constant.

Lemma 5.6 ([24]) Let A be a Banach algebra with a unit e, $a, b \in$ A. If a commutes with $b$, then

$$
\begin{aligned}
& \rho(a+b) \leq \rho(a)+\rho(b), \\
& \rho(a b) \leq \rho(a) \rho(b) .
\end{aligned}
$$

Lemma 5.7 ([28]) If $E$ is a real Banach space with a solid cone $P$

(1) If $a, b, c \in E$ and $a \leq b \ll c$, then $a \ll c$.

(2) If $a \in P$ and $a \ll c$ for each $c \gg \theta$, then $a=\theta$.

Lemma 5.8 ([16]) Let $P$ be a solid cone in a Banach algebra A. Suppose that $k \in P$ and $\left\{u_{n}\right\}$ is a c-sequence in $P$. Then $\left\{k u_{n}\right\}$ is a c-sequence.

Lemma 5.9 ([28]) Let $A$ be a Banach algebra with a unit e and $k \in A$. If $\lambda$ is a complex constant and $\rho(k)<|\lambda|$, then

$$
\rho\left((\lambda e-k)^{-1}\right) \leq \frac{1}{|\lambda|-\rho(k)} .
$$

Lemma 5.10 ([28]) Let $A$ be a Banach algebra with a unit e and $P$ be a solid cone in A. Let $a, k, l \in P$ hold $l \preccurlyeq k$ and $a \preccurlyeq l a$. If $\rho(k)<1$, then $a=\theta$.

\section{Applications to fixed point theory}

In this section, we prove some famous fixed point theorems satisfying generalized Lipschitz maps in the framework of $F$-cone metric space over Banach algebra $A$.

Theorem 6.1 Let $(X, F)$ be a $\theta$-complete F-cone metric space over Banach algebra $A$ and suppose that $T: X \rightarrow X$ is a mapping satisfying the following condition:

$$
F(T x, T x, T y) \preccurlyeq k F(x, x, y),
$$

where $\rho(k)<1$. Then $T$ has a unique fixed point. For each $x \in X$, the sequence of iterates $\left\{T^{n} x\right\}_{n \geq 1}$ converges to the fixed point. 
Proof For each $x_{0} \in X$ and $n \geq 1$, set $x_{1}=T x_{0}$ and $x_{n+1}=T^{n+1} x_{0}$. Then

$$
\begin{aligned}
F\left(x_{n}, x_{n}, x_{n+1}\right) & =F\left(T x_{n-1}, T x_{n-1}, T x_{n}\right) \\
& \preccurlyeq k F\left(x_{n-1}, x_{n-1}, x_{n}\right) \\
& \preccurlyeq k^{2} F\left(x_{n-2}, x_{n-2}, x_{n-1}\right) \\
& \vdots \\
& \preccurlyeq k^{n} F\left(x_{0}, x_{0}, x_{1}\right) .
\end{aligned}
$$

So, for $m>n$,

$$
\begin{aligned}
F\left(x_{n}, x_{n}, x_{m}\right) \preccurlyeq & s\left[F\left(x_{n}, x_{n}, x_{n+1}\right)+F\left(x_{n}, x_{n}, x_{n+1}\right)+F\left(x_{m}, x_{m}, x_{n+1}\right)-F\left(x_{n+1}, x_{n+1}, x_{n+1}\right)\right] \\
\preccurlyeq & s\left[2 F\left(x_{n}, x_{n}, x_{n+1}\right)+F\left(x_{n+1}, x_{n+1}, x_{m}\right)\right] \\
= & 2 s F\left(x_{n}, x_{n}, x_{n+1}\right)+s F\left(x_{n+1}, x_{n+1}, x_{m}\right) \\
\preccurlyeq & 2 s F\left(x_{n}, x_{n}, x_{n+1}\right)+s^{2}\left[F\left(x_{n+1}, x_{n+1}, x_{n+2}\right)+F\left(x_{n+1}, x_{n+1}, x_{n+2}\right)\right. \\
& \left.+F\left(x_{m}, x_{m}, x_{n+2}\right)-F\left(x_{n+2}, x_{n+2}, x_{n+2}\right)\right] \\
\preccurlyeq & 2 s F\left(x_{n}, x_{n}, x_{n+1}\right)+2 s^{2} F\left(x_{n+1}, x_{n+1}, x_{n+2}\right)+s^{2} F\left(x_{n+2}, x_{n+2}, x_{m}\right) \\
\preccurlyeq & 2 s F\left(x_{n}, x_{n}, x_{n+1}\right)+2 s^{2} F\left(x_{n+1}, x_{n+1}, x_{n+2}\right)+2 s^{3} F\left(x_{n+2}, x_{n+2}, x_{n+3}\right) \\
& +---+2 s^{m-n} F\left(x_{m-1}, x_{m-1}, x_{m}\right) \\
\preccurlyeq & 2 s k^{n} F\left(x_{0}, x_{0}, x_{1}\right)+2 s^{2} k^{n+1} F\left(x_{0}, x_{0}, x_{1}\right)+2 s^{3} k^{n+2} F\left(x_{0}, x_{0}, x_{1}\right) \\
& +---+2 s^{m-n} k^{m-1} F\left(x_{0}, x_{0}, x_{1}\right) \\
\preccurlyeq & \left(2 s^{n} k^{n}+2 s^{n+1} k^{n+1}+2 s^{n+2} k^{n+2}+---+2 s^{m-1} k^{m-1}\right) F\left(x_{0}, x_{0}, x_{1}\right) \\
= & 2 s^{n} k^{n}\left[e+(s k)+(s k)^{2}+---+(s k)^{m-n-1}\right] F\left(x_{0}, x_{0}, x_{1}\right) \\
\preccurlyeq & 2(s k)^{n}(e-s k)^{-1} F\left(x_{0}, x_{0}, x_{1}\right) .
\end{aligned}
$$

By Remark 2.4, $\left\|(s k)^{n} \cdot F\left(x_{0}, x_{0}, x_{1}\right)\right\| \leq\left\|(s k)^{n}\right\|\left\|F\left(x_{0}, x_{0}, x_{1}\right)\right\| \rightarrow 0$. By Lemma 5.4, we have $\left\{(s k)^{n} F\left(x_{0}, x_{0}, x_{1}\right)\right\}$ is a $c$-sequence. Next, by using Lemmas 5.7 and 5.8 , we conclude that $\left\{x_{n}\right\}$ is a $\theta$-Cauchy sequence in $X$.

By the $\theta$-completeness of $X$, there exists $u \in X$ such that

$$
\begin{aligned}
\lim _{n \rightarrow \infty} F\left(x_{n}, x_{n}, u\right) & =\lim _{n \rightarrow \infty} F\left(x_{n}, x_{n}, x_{m}\right) \\
& =F(u, u, u)=\theta .
\end{aligned}
$$

Furthermore, one has

$$
\begin{aligned}
F(u, u, T u) & \preccurlyeq s\left[F\left(u, u, T x_{n}\right)+F\left(u, u, T x_{n}\right)+F\left(T u, T u, T x_{n}\right)-F\left(T x_{n}, T x_{n}, T x_{n}\right)\right] \\
& \preccurlyeq s\left[2 F\left(u, u, T x_{n}\right)+F\left(T u, T u, T x_{n}\right)\right] \\
& =s\left[2 F\left(u, u, x_{n+1}\right)+F\left(u, u, x_{n}\right)\right] .
\end{aligned}
$$


Now that $\left\{F\left(u, u, x_{n+1}\right)\right\}$ and $\left\{F\left(u, u, x_{n}\right)\right\}$ are $c$-sequences, by using Lemmas 5.7 and 5.8, we conclude that $T u=u$. Thus $u$ is a fixed point of $T$.

Finally, we prove the uniqueness of the fixed point. In fact, if $v$ is another fixed point,

$$
\begin{aligned}
F(u, u, v) & =F(T u, T u, T v) \\
& \preccurlyeq k F(u, u, v) .
\end{aligned}
$$

That is,

$$
(e-k) F(u, u, v) \preccurlyeq \theta .
$$

Multiplying both sides above by

$$
(e-k)^{-1}=\sum_{i=0}^{\infty} k^{i} \geq 0,
$$

we get $F(u, u, v) \preccurlyeq \theta$. Thus, $F(u, u, v)=\theta$, which implies that $u=v$, a contradiction.

Hence, the fixed point is unique.

Corollary 6.2 Let $(X, F)$ be a $\theta$-complete F-cone metric space over Banach algebra A. Suppose that a mapping $T: X \rightarrow X$ satisfies, for some positive integer $n$,

$$
F\left(T^{n} x, T^{n} x, T^{n} y\right) \preccurlyeq k F(x, x, y)
$$

for all $x, y \in X$, where $k$ is a vector with $\rho(k)<\frac{1}{s}$. Then $T$ has a unique fixed point in $X$.

Proof From Theorem 6.1, $T^{n}$ has a unique fixed point $x^{*}$. But $T^{n}\left(T x^{*}\right)=T\left(T^{n} x^{*}\right)=T x^{*}$. So, $T x^{*}$ is also a fixed point of $T^{n}$. Hence $T x^{*}=x^{*}, x^{*}$ is a fixed point of $T$. Since the fixed point of $T$ is also a fixed point of $T^{n}$, then the fixed point of $T$ is unique.

We now prove Chatterjee's fixed point theorem in the new space.

Theorem 6.3 Let $(X, F)$ be a $\theta$-complete $F$-cone metric space over a Banach algebra $A$, and let $P$ be the underlying cone with $k \in P$ with $\rho(k)<\frac{1}{s+1}$. Suppose that a mapping $T: X \rightarrow X$ satisfies the generalized Lipschitz condition

$$
F(T x, T x, T y) \preccurlyeq k[F(T x, T x, x)+F(T y, T y, y)]
$$

for all $x, y \in X$. Then $T$ has a unique fixed point in $X$. And for any $x \in X$, the iterative sequence $\left\{T^{n} x\right\}$ converges to the fixed point.

Proof Let $x_{0} \in X$ be arbitrarily given and set $x_{n}=T^{n} x, n \geq 1$. We have

$$
\begin{aligned}
F\left(x_{n+1}, x_{n+1}, x_{n}\right) & =F\left(T x_{n}, T x_{n}, T x_{n-1}\right) \\
& \preccurlyeq k\left[F\left(T x_{n}, T x_{n}, x_{n}\right)+F\left(T x_{n-1}, T x_{n-1}, x_{n-1}\right)\right] \\
& \preccurlyeq k\left[F\left(x_{n+1}, x_{n+1}, x_{n}\right)+F\left(x_{n}, x_{n}, x_{n-1}\right)\right],
\end{aligned}
$$


which implies

$$
(e-k) F\left(x_{n+1}, x_{n+1}, x_{n}\right) \preccurlyeq k F\left(x_{n-1}, x_{n-1}, x_{n}\right) .
$$

Note that $\rho(k)<(s+1) \rho(k)<1$.

Then by Lemma 5.5 it follows that $(e-k)$ is invertible.

Multiplying both sides of (6.3.1) by $(e-k)^{-1}$, we get

$$
F\left(x_{n+1}, x_{n+1}, x_{n}\right) \preccurlyeq(e-k)^{-1} \cdot k F\left(x_{n-1}, x_{n-1}, x_{n}\right) .
$$

As is shown in the proof of Theorem 6.1, $\left\{x_{n}\right\}$ is a $\theta$-Cauchy sequence, and by the $\theta$ completeness of $X$, there exists $z \in X$ such that

$$
\begin{aligned}
\lim _{n \rightarrow \infty} F\left(x_{n}, x_{n}, z\right) & =\lim _{n, m \rightarrow \infty} F\left(x_{n}, x_{n}, x_{m}\right) \\
& =F(z, z, z) \\
& =\theta .
\end{aligned}
$$

Put $h=(e-k)^{-1} \cdot k, \therefore F\left(x_{n+1}, x_{n+1}, x_{n}\right) \preccurlyeq h F\left(x_{n-1}, x_{n-1}, x_{n}\right)$

$$
\begin{aligned}
\rho(h) & =\rho\left[(e-k)^{-1} \cdot k\right] \\
& \leq \rho(e-k)^{-1} \cdot \rho(k) \\
& \leq \frac{\rho(k)}{1-\rho(k)}<\frac{1}{s} .
\end{aligned}
$$

We shall show that $z$ is a fixed point of $T$.

We have

$$
\begin{aligned}
F(z, z, T z) & \preccurlyeq s\left[F\left(z, z, T x_{n}\right)+F\left(z, z, T x_{n}\right)+F\left(T z, T z, T x_{n}\right)-F\left(T x_{n}, T x_{n}, T x_{n}\right)\right] \\
& \preccurlyeq s\left[2 F\left(z, z, T x_{n}\right)+F\left(T z, T z, T x_{n}\right)\right] \\
& \preccurlyeq s\left[2 F\left(z, z, x_{n+1}\right)+k\left(F(T z, T z, z)+F\left(T x_{n}, T x_{n}, x_{n}\right)\right)\right] \\
& =2 s F\left(z, z, x_{n+1}\right)+\operatorname{sk} F(z, z, T z)+\operatorname{sk} F\left(x_{n+1}, x_{n+1}, x_{n}\right),
\end{aligned}
$$

which implies that

$$
(e-s k) F(z, z, T z) \preccurlyeq 2 s F\left(z, z, x_{n+1}\right)+\operatorname{sk} F\left(x_{n+1}, x_{n+1}, x_{n}\right) .
$$

Since $\rho(s k)<(s+1) \rho(k)<1$, it concludes by Lemma 5.5 that $(e-s k)$ is invertible. So,

$$
F(z, z, T z) \preccurlyeq(e-s k)^{-1} \cdot\left[2 s F\left(z, z, x_{n+1}\right)+s k F\left(x_{n+1}, x_{n+1}, x_{n}\right)\right] .
$$

Now that $\left\{F\left(z, z, x_{n+1}\right)\right\}$ and $\left\{F\left(x_{n+1}, x_{n+1}, x_{n}\right)\right\}$ are $c$-sequences, then by Lemmas 5.7 and 5.8 , it concludes that $T z=z$. Then $z$ is a fixed point of $T$. 
Finally, we prove the uniqueness of the fixed point. In fact, if $v$ is another fixed point, then

$$
\begin{aligned}
F(v, v, z) & =F(T v, T v, T z) \\
& \preccurlyeq k[F(T v, T v, v)+F(T z, T z, z)] \\
& =k[F(v, v, v)+F(z, z, z)] .
\end{aligned}
$$

So, $F(v, v, z) \preccurlyeq \theta$, which is a contradiction. Hence $F(v, v, z)=\theta$.

So, $v=z$.

Hence the fixed point is unique.

Example 6.4 Let the Banach algebra $A$ and the cone $P$ be the same ones as those in Example 3.3, and let $X=R^{+}$. Define a mapping $F: X^{3} \rightarrow A$ as in Example 3.3. We make a conclusion that $(X, F)$ is a $\theta$-complete $F$-cone metric space over Banach algebra $A$. Now define the mapping $T: X \rightarrow X$ by $T(x)=\cos \frac{x}{2}-1$.

Then

$$
\begin{aligned}
& F(T x, T x, T y)(t) \\
& =\left((\max \{T x, T y\})^{2}+(\max \{T x, T y\})^{2}, \alpha\left((\max \{T x, T y\})^{2}\right.\right. \\
& \left.\left.+(\max \{T x, T y\})^{2}\right)\right) e^{t} \\
& =2\left((\max \{T x, T y\})^{2}, \alpha(\max \{T x, T y\})^{2}\right) e^{t} \\
& =2\left(\left(\max \left\{\cos \frac{x}{2}-1, \cos \frac{y}{2}-1\right\}\right)^{2}, \alpha\left(\max \left\{\cos \frac{x}{2}-1, \cos \frac{y}{2}-1\right\}\right)^{2}\right) e^{t} \\
& <2\left(\left(\max \left\{\cos \frac{x}{2}, \cos \frac{y}{2}\right\}\right)^{2}, \alpha\left(\max \left\{\cos \frac{x}{2}, \cos \frac{y}{2}\right\}\right)^{2}\right) e^{t} \\
& \preccurlyeq 2\left(\left(\max \left\{\frac{x}{2}, \frac{y}{2}\right\}\right)^{2}, \alpha\left(\max \left\{\frac{x}{2}, \frac{y}{2}\right\}\right)^{2}\right) e^{t} \\
& =\frac{1}{4}\left[2(\max \{x, y\})^{2}, 2 \alpha(\max \{x, y\})^{2}\right] e^{t} \\
& =\frac{1}{4} F(x, x, y)(t) \text {, }
\end{aligned}
$$

where $k=\frac{1}{4}$, then all the conditions of Theorem 6.1 hold trivially good and 0 is the unique fixed point of $T$. Clearly, $T$ is a generalized Lipschitz map in $X$.

\section{Expansive mapping on $F$-cone metric space over Banach algebra}

In this section, we define expansive maps in $F$-cone metric spaces over Banach algebra.

Definition 7.1 Let $(X, F)$ be an $F$-cone metric space over Banach algebra $A$ and $P$ be a cone in $A$. A map $T: X \rightarrow X$ is said to be an expansive mapping where $k, k^{-1} \in P$ are called the generalized Lipschitz constants with $\rho\left(k^{-1}\right)<1$ for all $x, y \in X$ such that

$$
F(T x, T x, T y) \succcurlyeq k F(x, x, y) .
$$


Example 7.2 Let the Banach algebra $A$ and the cone $P$ be the same ones as those in Example 3.3, and let $X=R^{+}$. Define a mapping $F: X^{3} \rightarrow A$ as in Example 3.3. Then $(X, F)$ is an $F$-cone metric space over Banach algebra $A$. Now define the mapping $T: X \rightarrow X$ by $T(x)=2 x$. Then, for all $x, y \in X$, we have

$$
\begin{aligned}
F & (T x, T x, T y)(t) \\
& =\left((\max \{T x, T y\})^{2}+(\max \{T x, T y\})^{2}, \alpha\left((\max \{T x, T y\})^{2}+(\max \{T x, T y\})^{2}\right)\right) e^{t} \\
& =2\left((\max \{T x, T y\})^{2}, \alpha(\max \{T x, T y\})^{2}\right) e^{t} \\
& =2\left((\max \{2 x, 2 y\})^{2}, \alpha(\max \{2 x, 2 y\})^{2}\right) e^{t} \\
& =8\left((\max \{x, y\})^{2}, \alpha(\max \{x, y\})^{2}\right) e^{t} \\
& \succcurlyeq 4\left[\left((\max \{x, y\})^{2}+(\max \{x, y\})^{2}\right), \alpha\left((\max \{x, y\})^{2}+(\max \{x, y\})^{2}\right)\right] e^{t} \\
& \succcurlyeq 4 F(x, x, y)(t),
\end{aligned}
$$

where $k=4$. Clearly, $T$ is an expansive map in $X$.

Now we present a fixed point theorem for such maps.

Theorem 7.3 Let $(X, F)$ be a $\theta$-complete F-cone metric space over Banach algebra, and let $P$ be an underlying solid cone with $k \in P$ with $\rho\left((e+k+s k-a)(b+c-2 s k)^{-1}\right)<\frac{1}{s}$. Let $f$ and $g$ be two surjective selfmaps of $X$ satisfying

$$
F(f x, f x, g y)+k[F(x, x, g y)+F(y, y, f x)] \succcurlyeq a F(x, x, f x)+b F(y, y, g y)+c F(x, x, y)
$$

for all $x, y \in X$, and then $f$ and $g$ have a unique common fixed point in $X$.

Proof We define a sequence $x_{n}$ as follows for $n=0,1,2,3, \ldots$ :

$$
x_{2 n}=f x_{2 n+1}, \quad x_{2 n+1}=g x_{2 n+2} .
$$

If $x_{2 n}=x_{2 n+1}=x_{2 n+2}$ for some $n$, then we say that $x_{2 n}$ is a fixed point of $f$ and $g$. Therefore, we suppose that no two consecutive terms of the sequence $\left\{x_{n}\right\}$ are equal.

Now, putting $x=x_{2 n+1}$ and $y=x_{2 n+2}$ in (7.3.1), we get

$$
\begin{aligned}
& F\left(f x_{2 n+1}, f x_{2 n+1}, g x_{2 n+2}\right)+k\left[F\left(x_{2 n+1}, x_{2 n+1}, g x_{2 n+2}\right)+F\left(x_{2 n+2}, x_{2 n+2}, f x_{2 n+1}\right)\right] \\
& \quad \succcurlyeq a F\left(x_{2 n+1}, x_{2 n+1}, f x_{2 n+1}\right)+b F\left(x_{2 n+2}, x_{2 n+2}, g x_{2 n+2}\right)+c F\left(x_{2 n+1}, x_{2 n+1}, x_{2 n+2}\right), \\
& F\left(x_{2 n}, x_{2 n}, x_{2 n+1}\right)+k\left[F\left(x_{2 n+1}, x_{2 n+1}, x_{2 n+1}\right)+F\left(x_{2 n+2}, x_{2 n+2}, x_{2 n}\right)\right] \\
& \quad \succcurlyeq a F\left(x_{2 n+1}, x_{2 n+1}, x_{2 n}\right)+b F\left(x_{2 n+2}, x_{2 n+1}, x_{2 n+1}\right)+c F\left(x_{2 n+1}, x_{2 n+1}, x_{2 n+2}\right), \\
& a F\left(x_{2 n+1}, x_{2 n+1}, x_{2 n}\right)+b F\left(x_{2 n+2}, x_{2 n+1}, x_{2 n+1}\right)+c F\left(x_{2 n+1}, x_{2 n+1}, x_{2 n+2}\right) \\
& \quad \preccurlyeq F\left(x_{2 n}, x_{2 n}, x_{2 n+1}\right)+k\left[F\left(x_{2 n+1}, x_{2 n+1}, x_{2 n}\right)+s\left(F\left(x_{2 n+2}, x_{2 n+2}, x_{2 n+1}\right),\right.\right. \\
& \left.\left.\quad+F\left(x_{2 n+2}, x_{2 n+2}, x_{2 n+1}\right)+F\left(x_{2 n}, x_{2 n}, x_{2 n+1}\right)-F\left(x_{2 n+1}, x_{2 n+1}, x_{2 n+1}\right)\right)\right] \quad\left[\mathrm{By}\left(F_{2}\right)\right], \\
& a F\left(x_{2 n+1}, x_{2 n+1}, x_{2 n}\right)+b F\left(x_{2 n+2}, x_{2 n+1}, x_{2 n+1}\right)+c F\left(x_{2 n+1}, x_{2 n+1}, x_{2 n+2}\right)
\end{aligned}
$$




$$
\begin{aligned}
\preccurlyeq & F\left(x_{2 n}, x_{2 n}, x_{2 n+1}\right)+k F\left(x_{2 n+1}, x_{2 n+1}, x_{2 n}\right)+2 s k F\left(x_{2 n+2}, x_{2 n+2}, x_{2 n+1}\right) \\
& +\operatorname{sk} F\left(x_{2 n}, x_{2 n}, x_{2 n+1}\right)-s k F\left(x_{2 n}, x_{2 n}, x_{2 n+1}\right), \\
(b+c & -2 s k) F\left(x_{2 n+1}, x_{2 n+1}, x_{2 n+2}\right) \preccurlyeq(e+k+s k-a) F\left(x_{2 n}, x_{2 n}, x_{2 n+1}\right) .
\end{aligned}
$$

Put $b+c-2 s k=r$, then

$$
r F\left(x_{2 n+1}, x_{2 n+1}, x_{2 n+2}\right) \preccurlyeq(e+k+s k-a) F\left(x_{2 n}, x_{2 n}, x_{2 n+1}\right) .
$$

Since $r$ is invertible, to multiply $r^{-1}$ on both sides of (7.3.2),

$$
F\left(x_{2 n+1}, x_{2 n+1}, x_{2 n+2}\right) \preccurlyeq h F\left(x_{2 n}, x_{2 n}, x_{2 n+1}\right),
$$

where $h=(e+k+s k-a)(b+c-2 s k)^{-1}$.

Note that $\rho(h)<\frac{1}{s}$.

Hence by the proof of Theorem 6.1, we can easily see that the sequence $\left\{x_{n}\right\}$ is a $\theta$-Cauchy sequence. Moreover, by the $\theta$-completeness of $X$, there exists $x^{*} \in X$ such that

$$
\lim _{n \rightarrow \infty} F\left(x_{n}, x_{n}, x^{*}\right)=\lim _{n, m \rightarrow \infty} F\left(x_{n}, x_{n}, x_{m}\right)=F\left(x^{*}, x^{*}, x^{*}\right)=\theta
$$

Since $f$ and $g$ are surjective maps and hence there exist two points $y$ and $y^{\prime}$ in $X$ such that $x^{*}=f y$ and $x^{*}=g y^{\prime}$.

Consider

$$
\begin{aligned}
F\left(x_{2 n}, x_{2 n}, x^{*}\right)= & F\left(f x_{2 n+1}, f x_{2 n+1}, g y^{\prime}\right) \\
\succcurlyeq & -k\left[F\left(x_{2 n+1}, x_{2 n+1}, g y^{\prime}\right)+F\left(y^{\prime}, y^{\prime}, f x_{2 n+1}\right)\right]+a F\left(x_{2 n+1}, x_{2 n+1}, f x_{2 n+1}\right) \\
& +b F\left(y^{\prime}, y^{\prime}, g y^{\prime}\right)+c F\left(x_{2 n+1}, x_{2 n+1}, y^{\prime}\right) .
\end{aligned}
$$

Then

$$
\begin{aligned}
F\left(x_{2 n}, x_{2 n}, x^{*}\right) \succcurlyeq & -k F\left(x_{2 n+1}, x_{2 n+1}, x^{*}\right)-k F\left(y^{\prime}, y^{\prime}, x_{2 n}\right)+a F\left(x_{2 n+1}, x_{2 n+1}, x_{2 n}\right) \\
& +b F\left(y^{\prime}, y^{\prime}, x^{*}\right)+c F\left(x_{2 n+1}, x_{2 n+1}, y^{\prime}\right) .
\end{aligned}
$$

Since

$$
F\left(y^{\prime}, y^{\prime}, x^{*}\right) \preccurlyeq s\left[2 F\left(y^{\prime}, y^{\prime}, x_{2 n+1}\right)+F\left(x^{*}, x^{*}, x_{2 n+1}\right)-F\left(x_{2 n+1}, x_{2 n+1}, x_{2 n+1}\right)\right],
$$

so

$$
\begin{aligned}
- & k F\left(x_{2 n+1}, x_{2 n+1}, x^{*}\right)-k F\left(y^{\prime}, y^{\prime}, x_{2 n}\right)+a F\left(x_{2 n+1}, x_{2 n+1}, x_{2 n}\right)+c F\left(x_{2 n+1}, x_{2 n+1}, y^{\prime}\right) \\
& \preccurlyeq F\left(x_{2 n}, x_{2 n}, x^{*}\right)-b\left[2 s F\left(y^{\prime}, y^{\prime}, x_{2 n+1}\right)+s F\left(x^{*}, x^{*}, x_{2 n+1}\right)-s F\left(x_{2 n+1}, x_{2 n+1}, x_{2 n+1}\right)\right], \\
- & k F\left(x_{2 n+1}, x_{2 n+1}, x^{*}\right)+a F\left(x_{2 n+1}, x_{2 n+1}, x_{2 n}\right)+c F\left(x_{2 n+1}, x_{2 n+1}, y^{\prime}\right) \\
& \preccurlyeq k s\left[2 F\left(x_{2 n}, x_{2 n}, x_{2 n+1}\right)+F\left(x_{2 n+1}, x_{2 n+1}, y^{\prime}\right)-F\left(x_{2 n+1}, x_{2 n+1}, x_{2 n+1}\right)\right]
\end{aligned}
$$




$$
\begin{aligned}
& +s\left[2 F\left(x_{2 n}, x_{2 n}, x_{2 n+1}\right)+F\left(x_{2 n+1}, x_{2 n+1}, x^{*}\right)\right] \\
& -b\left[2 s F\left(y^{\prime}, y^{\prime}, x_{2 n+1}\right)+s F\left(x^{*}, x^{*}, x_{2 n+1}\right)-F\left(x_{2 n+1}, x_{2 n+1}, x_{2 n+1}\right)\right]
\end{aligned}
$$

which implies

$$
\begin{aligned}
& (2 s b+c-s k) F\left(x_{2 n+1}, x_{2 n+1}, y^{\prime}\right) \\
& \quad \preccurlyeq(s-b s+k) F\left(x_{2 n+1}, x_{2 n+1}, x^{*}\right)+(2 s k+2 s-a) F\left(x_{2 n+1}, x_{2 n+1}, x_{2 n}\right) .
\end{aligned}
$$

Since $2 s b+c-s k=r$ is invertible, we have

$$
\begin{aligned}
& r F\left(x_{2 n+1}, x_{2 n+1}, y^{\prime}\right) \\
& \quad \preccurlyeq(s-b s+k) \cdot F\left(x_{2 n+1}, x_{2 n+1}, x^{*}\right)+(2 s k+2 s-a) \cdot F\left(x_{2 n+1}, x_{2 n+1}, x_{2 n}\right), \\
& F\left(x_{2 n+1}, x_{2 n+1}, y^{\prime}\right) \\
& \quad \preccurlyeq r^{-1}\left\{(s-b s+k) F\left(x_{2 n+1}, x_{2 n+1}, x^{*}\right)+(2 s k+2 s-a) F\left(x_{2 n+1}, x_{2 n+1}, x_{2 n}\right)\right\} .
\end{aligned}
$$

Now that $\left\{F\left(x_{2 n+1}, x_{2 n+1}, x^{*}\right)\right\}$ and $\left\{F\left(x_{2 n+1}, x_{2 n+1}, x_{2 n}\right)\right\}$ are $c$-sequences, then by using Lemmas 5.7 and 5.8, we conclude that $g x_{n+2}=y^{\prime}$.

Finally, we prove the uniqueness of the fixed point. In fact, if $y^{*}$ is another common fixed point of $f$ and $g$, that is, $f y^{*}=y^{*}$ and $g y^{*}=y^{*}$,

$$
\begin{aligned}
& F\left(x, x, y^{*}\right) \\
& =F\left(T x, T x, T y^{*}\right) \\
& \succcurlyeq-k\left[F\left(x, x, g y^{*}\right)+F\left(y^{*}, y^{*}, f x\right)\right]+a F(x, x, f x)+b F\left(y^{*}, y^{*}, g y^{*}\right)+c F\left(x, x, y^{*}\right) \\
& \Rightarrow \quad F\left(x, x, y^{*}\right) \succcurlyeq-k\left[F\left(x, x, y^{*}\right)+F\left(y^{*}, y^{*}, x\right)\right]+a F(x, x, x)+b F\left(y^{*}, y^{*}, y^{*}\right) \\
& +c F\left(x, x, y^{*}\right) \\
& \Rightarrow \quad F\left(x, x, y^{*}\right) \succcurlyeq(-2 k+c) F\left(x, x, y^{*}\right)
\end{aligned}
$$

or

$$
\begin{aligned}
& (c-2 k) F\left(x, x, y^{*}\right) \preccurlyeq F\left(x, x, y^{*}\right), \\
& (c-2 k-e) F\left(x, x, y^{*}\right) \preccurlyeq \theta,
\end{aligned}
$$

which means $F\left(x, x, y^{*}\right)=\theta$, which implies that $x=y^{*}$, a contradiction. Hence the fixed point is unique.

Corollary 7.4 Let $(X, F)$ be a $\theta$-complete F-cone metric space over Banach algebra, and let $P$ be an underlying solid cone, where $c \in P$ is a generalized Lipschitz constant with $\rho(c)^{-1}<\frac{1}{s}$. Let $f$ and $g$ be two surjective selfmaps of $X$ satisfying

$$
F(f x, f x, g y) \succcurlyeq c F(x, x, y)
$$

Then $f$ and $g$ have a unique common fixed point in X. 
Proof If we put $k, a, b=\theta$ in Theorem 7.3, then we get the above Corollary 7.4.

Corollary 7.5 Let $(X, F)$ be a $\theta$-complete F-cone metric space over Banach algebra, and let $P$ be an underlying solid cone, where $c \in P$ is a generalized Lipschitz constant with $\rho(c)^{-1}<\frac{1}{s}$. Let $f$ be a surjective selfmap of $X$ satisfying

$$
F(f x, f x, f y) \succcurlyeq c F(x, x, y)
$$

Then $f$ has a unique fixed point in $X$.

Proof If we put $f=g$ in Corollary 7.4, then we get the above Corollary 7.5 which is an extension of Theorem 1 of Wang et al. [29] in an $F$-cone metric space over Banach algebra.

Corollary 7.6 Let $(X, F)$ be a $\theta$-complete F-cone metric space over Banach algebra, and let $P$ be an underlying solid cone, where $c \in P$ is a generalized Lipschitz constant with $\rho(c)^{-1}<\frac{1}{s}$. Let $f$ be a surjective selfmap of $X$, and suppose that there exists a positive integer $n$ satisfying

$$
F\left(f^{n} x, f^{n} x, f^{n} y\right) \succcurlyeq c F(x, x, y) .
$$

Then $f$ has a unique fixed point in $X$.

Proof From Corollary $7.5 f^{n}$ has a unique fixed point $z$. But $f^{n}(f z)=f\left(f^{n} z\right)=f z$, so $f z$ is also a fixed point of $f^{n}$. Hence $f z=z, z$ is a fixed point of $f$. Since the fixed point of $f$ is also a fixed point of $f^{n}$, the fixed point of $f$ is unique.

Corollary 7.7 Let $(X, F)$ be a $\theta$-complete F-cone metric space over Banach algebra, and let $P$ be an underlying solid cone, where $a, b, c,-a \in P$ are generalized Lipschitz constants with $\rho\left[(e-a)(b+c)^{-1}\right]<\frac{1}{s}$. Let $f$ and $g$ be two surjective selfmaps of $X$ satisfying

$$
F(f x, f x, g y) \succcurlyeq a F(x, x, f x)+b F(y, y, g y)+c F(x, x, y) .
$$

Then $f$ and $g$ have a unique common fixed point in $X$.

Proof If we put $k=\theta$ in Theorem 7.3, then we get the above Corollary 7.7.

Corollary 7.8 Let $(X, F)$ be a $\theta$-complete F-cone metric space over Banach algebra, and let $P$ be an underlying solid cone, where $a, b, c,-a \in P$ are generalized Lipschitz constants with $\rho\left[(e-a)(b+c)^{-1}\right]<\frac{1}{s}$. Let $f$ be a surjective selfmap of $X$ satisfying

$$
F(f x, f x, f y) \succcurlyeq a F(x, x, f x)+b F(y, y, f y)+c F(x, x, y) .
$$

Then $f$ has a unique fixed point in $X$.

Proof If we put $f=g$ in Corollary 7.7, then we get the above Corollary 7.8 which is an extension of Theorem 2 of Wang et al. [29] in an F-cone metric space over Banach algebra. 


\section{Conclusion}

In this paper, we introduce an $F$-cone metric space over Banach algebra which generalizes an $N_{p}$-cone metric space over Banach algebra and an $N_{b}$-cone metric space over Banach algebra. We introduce the concept of generalized Lipschitz and expansive mapping in the new structure. Also we derive the existence and uniqueness of some fixed point theorems for such spaces. Our main theorems extend and unify the existing results in the recent literature. Example is constructed to support our result.

\section{Competing interests}

The authors declare that they have no competing interests.

\section{Authors' contributions}

All authors contributed equally to the writing of this paper. All authors read and approved the final manuscript.

\section{Author details}

'Department of Mathematics, NRI Institute of Information Science and Technology, Bhopal, (M.P.), 462021, India. ${ }^{2}$ Nonlinear Analysis Research Group, Ton Duc Thang University, Ho Chi Minh City, Vietnam. ${ }^{3}$ Faculty of Mathematics and Statistics, Ton Duc Thang University, Ho Chi Minh City, Vietnam. ${ }^{4}$ Department of Mathematics, Government M V M, Bhopal, (M.P.), 462001, India.

\section{Publisher's Note}

Springer Nature remains neutral with regard to jurisdictional claims in published maps and institutional affiliations.

Received: 23 December 2016 Accepted: 28 April 2017 Published online: 15 May 2017

\section{References}

1. Matthews, SG: Partial metric topology. Ann. N.Y. Acad. Sci. 728(1), 183-197 (1994)

2. Bakhtin, IA: The contraction mapping principle in quasi-metric spaces. In: Functional Analysis, vol. 30. Ulyanovsk. Gos. Ped. Inst, Ulyanovsk (1989)

3. Huang, LG, Zhang, X: Cone metric spaces and fixed point theorem for contractive mappings. J. Math. Anal. Appl. $332(2), 1468-1476(2007)$

4. Rezapour, S, Hamalbarani, R: Some notes on the paper cone metric space and fixed point theorems of contractive mappings. J. Math. Anal. Appl. 345, 719-724 (2008)

5. Kadelburg, Z, Radenović, S, Rakočević, V: A note on the equivalence of some metric and cone metric fixed point results. Appl. Math. Lett. 24, 370-374 (2011)

6. Fernandez, J, Malviya, N, Fisher, B: The asymptotically regularity and sequences in partial cone $b$-metric spaces with application. Filomat 30(10), 2749-2760 (2016). doi:10.2298/FIL1610749F

7. Fernandez, J, Modi, G, Malviya, N: Some fixed point theorems for contractive maps in N-cone metric spaces. Math. Sci. 9, 33-38 (2015). doi:10.1007/s40096-015-0145-x

8. Fernandez, J, Saxena, K, Malviya, N: Fixed points of expansive maps in partial cone metric spaces. Gazi Univ. J. Sci. 27(4), 1085-1091 (2014)

9. Fernandez, J, Saxena, K, Malviya, N: The asymptotically regularity of maps and sequences in partial cone metric spaces with application. J. Pure Math. 31, 1-12 (2014)

10. Malviya, $N$, Fisher, B: $N$-cone metric space and fixed points of asymptotically regular maps. Filomat J. Math. (preprint)

11. Ismat, B: Abbas Mujahid and Nazir Talat: generalized cone metric spaces. J. Nonlinear Sci. Appl. 3, 21-31 (2010)

12. Aage, CT, Salunke, JN: Some fixed point theorem in generalized $D^{*}$-metric spaces. Appl. Sci. 12, 1-13 (2010)

13. Malviya, N, Chouhan, P: Fixed point of expansive type mapping in N-cone metric space. Math. Theory Model. 3(7), 81-86 (2013)

14. Du, WS: A note on cone metric fixed point theory and its equivalence. Nonlinear Anal. 72(5), 2259-2261 (2010)

15. Liu, H, Xu, S: Cone metric spaces with Banach algebras and fixed point theorems of generalized Lipschitz mappings. Fixed Point Theory Appl. 2013, 320 (2013)

16. Xu, S, Radenović, S: Fixed point theorems of generalized Lipschitz mappings on cone metric spaces over Banach algebras without assumption of normality. Fixed Point Theory Appl. 2014, 102 (2014)

17. Fernandez, J, Saxena, K, Malviya, N: On cone b2-metric spaces over Banach algebra, São. São Paulo J. Math. Sci. (accepted)

18. Fernandez, J, Malviya, N, Shukla, S: Cone b-metric like spaces over Banach algebra and fixed point theorems with application. Asian J. Math. Comput. Res. (accepted)

19. Fernandez, J, Saelee, S, Saxena, K, Malviya, N, Kumam, P: The A-cone metric space over Banach algebra with application of generalized Lipschitz and expansive maps in fixed point theory and integral equations. Cogent Math. 4, 1282690 (2017)

20. Fernandez, J: Partial cone metric spaces over Banach algebra and Generalized Lipschitz mappings with applications, Selected for Young Scientist Award 2016 M.P., India (accepted)

21. Fernandez, J, Saxena, K, Malviya, N: The $N_{p}$-cone metric space over Banach algebra and some fixed point theorems (communicated)

22. Fernandez, J, Saxena, K, Modi, G: The N-cone metric space over Banach algebra and some fixed point theorems (communicated) 
23. Fernandez, J, Saxena, K, Malviya, N: The $N_{b}$-cone metric space over Banach algebra (communicated)

24. Rudin, W: Functional Analysis, 2nd edn. McGraw-Hill, New York (1991)

25. Radenović, S, Rhoades, BE: Fixed point theorem for two non-self mappings in cone metric spaces. Comput. Math. Appl. 57, 1701-1707 (2009)

26. Aydi, H, Karapınar, E, Mustafa, Z: Coupled coincidence point results on generalized distance in ordered cone metric spaces. Positivity 17(4), 979-993 (2013)

27. Kadelburg, Z, Radenovic, S: A note on various types of cones and fixed point results in cone metric spaces. Asian J. Math. Appl. 2013, Article ID ama0104 (2013)

28. Huang, H, Radenović, S: Common fixed point theorems of generalized Lipschitz mappings in cone metric spaces over Banach algebras. Appl. Math. Inf. Sci. 9(6), 2983-2990 (2015)

29. Wang, SZ, Li, BY, Gao, ZM, Iseki, K: Some fixed point theorems for expansion mappings. Math. Jpn. 29, 631-636 (1984)

Submit your manuscript to a SpringerOpen ${ }^{\odot}$ journal and benefit from:

- Convenient online submission

- Rigorous peer review

- Immediate publication on acceptance

- Open access: articles freely available online

- High visibility within the field

- Retaining the copyright to your article 